\title{
Set-membership computation of integrals with uncertain endpoints
}

\author{
Olivier Mullier, Julien Alexandre dit Sandretto \\ ENSTA ParisTech, 828 bd des maréchaux 91120 Palaiseau, France \\ mullier@ensta.fr, alexandre@ensta.fr
}

Keywords. Integral; Set-membership Computation; Interval Methods.

Numerical integration is one of the fundamental tool of scientific computation. Providing a reliable result to such problem is important for validated simulation [1] or for global optimization with continuous objective function [2]. An important work on inclusion methods for integral equations can be found in [3]. We propose an efficient guaranteed method for the computation of the integral of a nonlinear continuous function $f$ between two interval endpoints $\left[x_{1}\right]$ and $\left[x_{2}\right]$, define by:

$$
\int_{\left[x_{1}\right]}^{\left[x_{2}\right]} f(x) d x=\left\{\int_{x_{1}}^{x_{2}} f(x) d x: x_{1} \in\left[x_{1}\right], x_{2} \in\left[x_{2}\right]\right\}
$$

Two cases can occur whether the intervals $\left[x_{1}\right]$ and $\left[x_{2}\right]$ intersect or not. If the two intervals do not intersect, we produce an outer approximation of the set of possible values for the integral proving the following proposed theorem:

Theorem 1. Let $f: \mathbb{R} \rightarrow \mathbb{R}$ be a differentiable function. For $\left[x_{1}\right]=\left[\underline{x_{1}}, \overline{x_{1}}\right]$ and $\left[x_{2}\right]=\left[x_{2}, \overline{x_{2}}\right]$ two intervals in $\mathbb{I}$. Then the minimum and the maximum of set (1) can be defined by

$$
\int_{\left[x_{1}\right]}^{\left[x_{2}\right]} f(x) d x=\left[\min \int_{\mathcal{X}_{1-}^{*} \cup\left\{\overline{x_{1}}\right\}}^{\mathcal{X}_{2+}^{*} \cup\left\{\underline{\left.x_{2}\right\}}\right.} f(x) d x, \max \int_{\mathcal{X}_{1+}^{*} \cup\left\{\overline{x_{1}}\right\}}^{\mathcal{X}_{2}^{*} \cup\left\{\underline{\left.x_{2}\right\}}\right.} f(x) d x\right]
$$

using

$\mathcal{X}_{i-}^{*}=\left\{x \in\left[x_{i}\right]: f(x)=0, f^{\prime}(x)<0\right\}, \mathcal{X}_{i+}^{*}=\left\{x \in\left[x_{i}\right]: f(x)=0, f^{\prime}(x)>0\right\}$.

When the two intervals intersect, Second Fundamental Theorem of Calculus is applied. An implementation of the computation of the bounds (2) is introduced using interval analysis [4] and tested on several examples.

\section{References}

[1] Alexandre dit Sandretto, J. and Chapoutot, A. (2016) Validated Explicit and Implicit Runge-Kutta Methods. Reliable Computing.

[2] Hansen, Eldon R. (2003) Global Optimization Using Interval Analysis. CRC Press.

[3] Corliss, G. (1987) Computing narrow inclusions for definite integrals. Computerarithmetic (eds. E. Kaucher et al.), pp. 150-179. Wiley-Teubner, Stuttgart.

[4] Moore, Ramon E. (1966) Interval analysis. Prentice-Hall Englewood Cliffs, NJ. 